\title{
The Neurological Significance of Abnormal Natural Killer Cell Activity in Chronic Toxigenic Mold Exposures
}

\author{
Ebere C. Anyanwu ${ }^{1}$, Andrew W. Campbell ${ }^{2}$, Joseph Jones ${ }^{3}$, and John Ehiri ${ }^{4}$ \\ ${ }^{1}$ Neurosciences Research, Cahers Inc., Conroe, TX; ${ }^{2}$ Medical Center for Immune, \\ Environmental, and Toxic Disorders, Spring, TX; ${ }^{3}$ Texas Southern University, Houston; \\ ${ }^{4}$ Department of Maternal and Child Health, School of Public Health, University of Alabama at \\ Birmingham \\ E-mail: ebereanyanwu@msn.com
}

Received September 29, 2003; Accepted October 10, 2003; Published November 13, 2003

Toxigenic mold activities produce metabolites that are either broad-spectrum antibiotics or mycotoxins that are cytotoxic. Indoor environmental exposure to these toxigenic molds leads to adverse health conditions with the main outcome measure of frequent neuroimmunologic and behavioral consequences. One of the immune system disorders found in patients presenting with toxigenic mold exposure is an abnormal natural killer cell activity. This paper presents an overview of the neurological significance of abnormal natural killer cell (NKC) activity in chronic toxigenic mold exposure. A comprehensive review of the literature was carried out to evaluate and assess the conditions under which the immune system could be dysfunctionally interfered with leading to abnormal NKC activity and the involvement of mycotoxins in these processes. The functions, mechanism, the factors that influence NKC activities, and the roles of mycotoxins in NKCs were cited wherever necessary. The major presentations are headache, general debilitating pains, nose bleeding, fevers with body temperatures up to $4^{\circ} \mathrm{C}\left(104^{\circ} \mathrm{F}\right)$, cough, memory loss, depression, mood swings, sleep disturbances, anxiety, chronic fatigue, vertigo/dizziness, and in some cases, seizures. Although sleep is commonly considered a restorative process that is important for the proper functioning of the immune system, it could be disturbed by mycotoxins. Most likely, mycotoxins exert some rigorous effects on the circadian rhythmic processes resulting in sleep deprivation to which an acute and transient increase in NKC activity is observed. Depression, psychological stress, tissue injuries, malignancies, carcinogenesis, chronic fatigue syndrome, and experimental allergic encephalomyelitis could be induced at very low physiological concentrations by mycotoxin-induced NKC activity. In the light of this review, it is concluded that chronic exposures to toxigenic mold could lead to abnormal NKC activity with a wide range of neurological consequences, some of which were headache, general debilitating pains, fever, cough, memory loss, depression, mood swings, sleep disturbances, anxiety, chronic fatigue, and seizures.

KEYWORDS: toxigenic mold, mycotoxins, neuroimmune disorders, NK cells, public health, United States 
DOMAINS: child health and human development, medical care, behavioral psychology, clinical psychology, nursing, toxicology

\section{INTRODUCTION}

Patients with chronic exposures to environmental toxigenic molds manifest adverse health reactions, some of which are allergic in nature, while others are pathologically systemic. Although these health conditions are probably inter-related, the manifestation of immune mechanism disorders is one of the underlying factors to which the neurological significance of the effects is the object of the discourse. The metabolism of toxigenic molds is known to produce two major metabolites: one that is beneficial (antibiotic) to humans and animals, while the other is nonbeneficial (mycotoxins). Mycotoxins have been shown to cause several immunotoxicologically undesired effects on humans, especially in the anatomical and chemical alterations of cell functions.

Hence, in various published studies, it is reported that patients presenting with toxigenic mold exposures show immune system dysfunctions to which abnormal natural killer cells (NKCs) are consistently observed. NKCs are a group of peripheral lymphocytes that contain a population of large granular lymphocytes. They are called "natural killer cells" because of their propensity to exert cytotoxic effects on various antigenic targets. They do not express T- or B-cell markers; however, they consistently express fragment crystalline (Fc) receptors on the cell surface[1]. The association of mycotoxins with various adverse health effects cannot be defined by the tests of abnormalities in the immune system alone. This is because of the possibility of underlying medical history and individual constitution. Furthermore, the reproducibility of immunologic test abnormalities reported under these conditions has not been verified with any degree of confidence in terms of scientific methodology. It is very important that one understands the biologic variability and diurnal variations in lymphoid cell numbers in interpreting cellular immunologic profiles to achieve diagnostic accuracy.

The aims of this paper were to review and put together the pieces of evidence scattered in the literature that might help to fill some of the information gap, and to evaluate the neurological significance of abnormal NKC activity in patients presenting with chronic toxigenic mold exposure.

\section{METHODS}

A comprehensive review of the literature was carried out to evaluate and assess the conditions under which the immune system could be dysfunctionally disrupted by chronic exposures to toxigenic molds. The functions, mechanism, the factors that influence NKC activities, and the roles of mycotoxins in NKC abnormalities was cited with pieces of evidence from the literature wherever necessary. This background led to the overall assessment, evaluation, and discussion of how chronic toxigenic mold exposures could influence the activity of NKCs.

\section{RESULTS OF REVIEW}

The major presentations common to patients with chronic toxigenic mold exposures were headache, general debilitating pains, nose bleeding, fevers with body temperatures up to $40^{\circ} \mathrm{C}$ $\left(104^{\circ} \mathrm{F}\right)$, cough, memory loss, depression, mood swings, sleep disturbances, anxiety, chronic fatigue, vertigo/dizziness, and in some cases, seizures. Although sleep is commonly considered a 
restorative process that is important for the proper functioning of the immune system, its deprivation was commonly reported in the literature. It is most likely that mycotoxins exert some rigorous effects on the circadian rhythmic processes resulting in sleep deprivation to which an acute and transient increase in NKC activity is observed.

Several data on the effects of mycotoxins including subjective and objective report assessments implicated sleep in the modulation of natural immunity and correlated with a reduction of NKC activity in major depression. Psychological stress had an influence on NKC activity, which played a central role in the protection against microbial infection and cancer. Anxiety was a typical type of psychological stress observed in patients with toxigenic mold exposures and was probably associated with mycotoxic activities. Transitory changes in NKCs associated with pain and stress were implicated in the development of infectious disease episodes after an acute stressful event. Although some studies suggested that acute severe pain without tissue injury markedly increased NKC cytotoxicity, there were some indications that patients presenting with chronic toxigenic mold exposures were likely to have tissue injuries due to mycotoxicity. It was also found that at very low physiological concentrations, mycotoxins significantly stimulated in vitro the antibody-dependent cellular cytotoxicity and NKC activities.

\section{DISCUSSION}

\section{Functions of NKC}

NKC activities are modulated by lymphokines and their proliferative and cytolytic activities are stimulated by interleukin-2 (IL-2) and enhanced by interferon (IFN)[1,2,3]. Combined depletions of NKCs and CD4(+) completely eliminated the antitumor effects. Also, NKCs play an important role in the activation of the potent immunotherapeutic effects elicited by intratumoral injections of IL-12 secreting dendritic cells (DCs) that are dependent on IL-12[4]. Although NKCs and DCs are two distinct cell types of innate immunity, in vitro interaction of human NKCs with autologous DCs results in DC lysis and the contact-dependent interactions between activated human NKCs and immature DCs (iDCs) provide a "control switch" for the immune system[5].

Hence, at low NKC/DC ratios, this interaction dramatically amplifies DC responses, whereas at high ratios it completely turns off their responses. Specifically, culture of activated human NKCs with iDCs, at low NK/DC ratios (1:5), led to exponential increases in DC cytokine production, which were completely dependent on cell-to-cell contact[5]. DC maturation was also driven by cognate interactions with NKCs and maturation was dependent on endogenously produced TNF-alpha in the culture. At slightly higher NKC/DC ratios (5:1), inhibition of DC function was the dominant feature due to potent killing by the autologous NKCs[5]. Impaired activation of NKCs and lymphokine-activated KCs has a role in the development of hepatocellular carcinoma[6,7,8].

\section{Mechanism of NKC Activity}

One of the major mechanisms by which the immune response deals with foreign or abnormal cells is to damage or destroy them. Such immunologic cytotoxicity may lead to complete loss of viability of the target cells (cytolysis) or an inhibition of the ability of the cells to continue growing (cytostasis). Immunologic cytotoxicity is a principal mechanism by which the immune response copes with and often eliminates foreign materials or abnormal cells. Although it is accepted that the understanding of the mechanisms of NKC functions might lead to novel therapeutic strategies for the treatment of human disease, to date the mechanisms of human NKC activity have been poorly defined[9]. 
The fact that serotonergic systems can modulate cholinergic functions implies that both the cholinergic and serotonergic systems are necessary for eliciting the conditioned enhancement of NKC activity[10]. NKCs should no longer be thought of as direct cytotoxic killers alone as they clearly serve a critical role in cytokine production, which may be important to control cancer, infection, and fetal implantation. NKCs play a critical role in immune surveillance and cancer therapy. However, new insights into NKC biology have suggested major roles for NKCs in infection controls and uterine function[11,12].

\section{Effects of Mycotoxins on NKC Function}

Mycotoxins significantly stimulate the proliferative response of splenocytes, thymocytes, T-cells mitogen Concanavalin A (Con A), and suppress the mitogenic responses to both lipopolysaccharide and Con A in prenatally exposed animals[13]. This indicated that prenatal exposure to relatively low-dose mycotoxins might induce immunosuppression[12,13,14,15,16]. The immunologic effects of Fusarium mycotoxins (deoxynivalenol [DON], 3acetyldeoxynivalenol, fusarenon-X, T-2 toxin, zearalenone, alpha-zearalenol, beta-zearalenol, and nivalenol) on human peripheral blood mononuclear cells from different blood donors showed that $\mathrm{T}$-2 toxin, fusarenon $\mathrm{X}$, nivalenol, and deoxynivalenol exerted the highest immunosuppressing effect in vitro[17,18]. Mycotoxin-induced immunosuppression is manifested as depressed T- or B-lymphocyte activity. By the virtue of mycotoxin inhibition of NKC activity, the protection against tumor development may also be affected[18].

A study[19] of possible immunomodulative effects of aflatoxin B1 (AFB1) at concentrations lower than 5.10(-11) M or $0.05 \mathrm{ppb}$ and 10(-5) M, respectively, on NKCs from healthy volunteers showed that AFB1 has an immunosuppressive effect on NKC activity. Various mycotoxins, such as gliotoxin, have immunomodulating activity. They selectively affect certain lymphocyte subsets, possibly through the mechanism of apoptosis, which is increased in vivo as well as in vitro[20]. Contrary to inhibitory effects, other mycotoxins (such as the Patulin mycotoxin produced by many fungal species of the genera Penicillium, Aspergillus, and Bryssochamys) were shown to increase the NKC and monocyte counts by 30 and 24\%, respectively[21].

\section{Factors that Influence NKC Activities}

NKC activity is influenced by a variety of factors including stress, chemical exposure, infections, chronic fatigue syndrome, immune deficiencies, and cancer. In an increasing number of patients with various diseases, IL-2, IFN, and NKC cytoxic factors have been shown to enhance NKC activity. Therefore, enhancement of IL-2 production may be useful in reactivation of NKCs in patients with chronic toxigenic mold exposures. Therefore, alterations of NKC functions by mycotoxins could be involved in the neuroimmune mechanism of neurological deficits found among the patients. In addition, alterations of NKC cytotoxicity control and NKC-derived cytokine release in mycotoxicity could be involved in the neuroinflammatory mechanism related to the progression of neurodegeneration in mycotoxicity. NKC activity has been associated with altered immunity and reduced occurrence of autoimmune diseases and malignancies.

It appears that an abnormal immunoregulatory response based on environmental mycotoxic damage to $\mathrm{T}$ cells is fundamental to the production of symptoms of mycotic illnesses[22]. That means that the stimulation of $\mathrm{T}$ cells or T-cell phenotypic subsets by environmental mycotoxins results in release of cytokines that can effect appropriate target cells of multiple organ systems, resulting in a wide range of neurological and behavioral symptoms[23]. It has been previously reported[24] that physiologic quantities of platelet-derived growth factor (PDGF) on their surface significantly inhibit human NKC cytotoxicity, and that patients with myelofibrosis and essential 
thrombocythemia have significantly elevated circulating levels of plasma PDGF. Pretreatment of normal NKCs with concentrated PDGF-containing, platelet-poor plasma from patients with these diseases significantly inhibits effects on the correlation between abnormal platelet release, plasma accumulation of PDGF, and the observed NKC immunodeficiency in these myeloproliferative patients if exposed to toxigenic molds.

\section{Neurological Significance and Sleep Disturbances}

Based on the literature reviews, the major presentations in patients with chronic toxigenic mold exposures were headache, general debilitating pains, nose bleeding, fevers, cough, memory loss, depression, mood swings, sleep disturbances, anxiety, chronic fatigue, vertigo/dizziness, and in some cases, seizures. The effects of sleep deprivation on immune profile in human have been reported in the literature[25,26,27]. However, since it is accepted that sleep deprivation modifies different host defense activities and increases the susceptibility to various diseases, it is most likely that mycotoxins exert some marked effects on the circadian rhythmic process resulting in sleep deprivation to which an acute and transient increase in NKC activity is observed. In addition to immune dysfunction, sleep disturbances due to mycotoxins may increase hypothalamic-pituitary-adrenocortical (HPA) axis activity in depression[26]. Since sleep is restoratively important for the proper functioning of the immune system, it could be disturbed by mycotoxic activity.

Moreover, in view of basic evidence of a reciprocal interaction between sleep and cytokines and the correlation with declines in NKC cytotoxicity activity[28], the mycotoxic effects may increase the severity of depressive symptoms or the presence of a mood disorder[29]. This suggestion is supported by several data measured by either subjective report or electroencephalographic (EEG) assessment that correlated the reduction of NKC activity with major depression[28,29,30].

\section{Psychological Stress}

Mycotoxicity can lead to psychological stress, and psychological stress has an influence on NKC activity, which plays a central role in protection against microbial infections and cancer. Anxiety is a typical psychological stress reported in a majority of chronic toxigenic mold exposures and anxiety and is associated with mycotoxic activities[31].

Transitory changes in NKCs associated with pain and stress were implicated in the development of infectious disease episodes after an acute stressful event[32]. Although acute severe pain without tissue injury markedly increased NKC cytotoxicity[33], indications in the review seemed to imply that patients presenting with chronic toxigenic mold exposures were more likely to have tissue injuries due to mycotoxicity. Hence, it was reported that mycotoxin in physiological concentrations ranging from $10(-11) \mathrm{M}$ to $10(-9) \mathrm{M}$ significantly stimulated in vitro the antibody-dependent cellular cytotoxicity and NKC activities in mouse leukocytes from axillary nodes, spleen, and thymus. It was also reported that mycotoxins induced change in IL-2 production, a rapid, transient and significant decrease of intracellular cyclic AMP at $30 \mathrm{~s}$, inositol 1,4,5-trisphosphate levels at 30 and $60 \mathrm{~s}$, and a stimulation of protein kinase C activity in membrane fractions after 5 min incubation, thus suggesting that inositol-phospholipid signaling and cAMP messenger systems are involved in the increase of mycotoxin-related NKC activity when leukocytes were incubated in the presence of mycotoxins (FB1)[33]. 


\section{Rules of Mycotoxins/NKC in Carcinogenesis}

The combination of mycotoxins, e.g., fumonisin (FB1) and aflatoxin (AFB1), showed synergistic toxicological and carcinogenic effects in a short-term carcinogenesis model in the liver of experimental animals. In this example, both the AFB1 and FB1 compounds exhibited slow cancer initiating potency individually, however, in combination, the number and size of GSTP(+) lesions significantly increased as compared to the separate treatments. This combination could pose an increased risk in humans[34]. AFB1 is one of the most potent rodent and human liver carcinogens and on cytochrome P450-specific metabolism, it induces mutation as well as mitotic recombination events in vitro systems hence, supporting the hypothesis that mitotic recombination represents a central mechanism of action in AFB1-induced liver carcinogenesis[35,36,37]. The NKC activity may facilitate the development of malignant tumors such as lymphoma cells[38]. A monoclonal antibody recognizing a NKC-associated antigen was used to sequentially quantify numbers of peripheral blood NKCs in a small group of patients with large cell lymphoma[39]. A role for NKCs in the surveillance and control of abnormal lymphoproliferation was suggested, as was the possible prognostic utility of sequential peripheral blood NKC quantification in patients with large cell lymphoma[39,40].

\section{The Mechanism of Mycotoxic Carcinogenesis}

The ubiquitous mycotoxin ochratoxin A (OTA), for example, is associated with the development of urothelial tumors and nephropathies in laboratory animals and in humans with stark species and sex differences with respect to susceptibility in disease development. The mechanism of action is probably by OTA-mediated disruptions in normal cell-cycle control and could be a major constituent of the mechanisms underlying both its carcinogenic and nephropathy-inducing activities[43]. Mycotoxins, especially OTA, specifically inhibit NKC activity and increase the growth of transplantable tumor cells without altering T-cell- or macrophage-mediated antitumor activity. Thus, ochratoxin appears to suppress NKC activity by inhibiting productions of basal interferon, thus suggesting a possible role for altered NKC function in the development of mycotoxin-induced carcinogenesis[14].

FB1 is epidemiologically linked to the high incidence of human esophageal cancer in South Africa and China and the mechanism is by inhibition of ceramide synthase and disruption of membrane phospholipids. The mechanism is also by the activation of protein kinase B which leads to increased survival, inhibition of GSK-3beta activity, and post-translational stabilization of cyclin D1, all events responsible for disruption of the cell cycle G (1)/S restriction point in hepatocytes[44,45]. Multiple signal transduction pathways such as PKC have been shown to play an important role in carcinogenesis. FB1 induces protein kinase $\mathrm{C}$ translocation via direct interaction with diacylglycerol site that also binds phorbol esters. Because phorbol esters are well-known tumor promoters, it is a more plausible cellular mechanism to explain the carcinogenicity of FB1[46,47]. Other studies suggested that the concept that FB1-induced mitogenesis might be the mechanism of carcinogenesis and that the primary target organs are the kidney and liver[48]. FB1 is also hepatocarcinogenic in rats, but epidemiological with esophageal cancer in humans[48,49].

\section{Mycotoxins, NKCs, and Chronic Fatigue Syndrome}

Several reports showed that chronic fatigue was one of the characteristic manifestations of chronic toxigenic mold exposures. Chronic fatigue syndrome (CFS) is characterized by unexplained, debilitating fatigue or easy fatigability lasting longer than 6 months. While a viral 
basis of infection is proposed to be the cause of CFS, other viral infections do not generally persist after several weeks. It is now acknowledged that mycotoxicity leads to immunologic disorders including abnormal functions and distributions of $\mathrm{T}$ lymphocytes, B lymphocytes, NKCs, and monocyte/macrophages, and these disorders have been described in CFS. NKCs are known to play an important role in host resistance against fungal and viral infection as well as in the regulation of the immune systems[50].

\section{NKCs in Malignancies}

NKCs are the subjects of great current interest because of their possible (in vivo) role in tumor cell surveillance and killing, and because of the potential application of cytokine-modulated NKCs in cancer immunotherapy. In addition, clonal proliferations of NKC-associated populations represent a high proportion of chronic (non-B) lymphoid malignancies and abnormal (both clonal and nonclonal) NKC-associated components are being increasingly reported in association with diverse clinical pictures such as autoimmune disease. This communication extensively reviews what is presently known regarding normal and leukemic NKC-associated phenotypic diversity, the mechanisms of NKC-mediated cytolysis, the role of NKCs in malignancy, and the diagnostic and cellular aspects of malignant NKC-associated proliferations[51]. Abnormal large granular lymphocytes appeared in peripheral blood and increased up to 17,000/microliters that had abundant pale cytoplasm with rich large azurophilic granules and a large nucleus with a few nucleoli. These cells did not have the activity of antibody-dependent cellular cytotoxicity but had natural killer activity[52].

Immunohistochemically, biopsy specimens taken from the lesional skin demonstrated that the NKC phenotype plays a role in the development of the abnormal skin reactions to mosquito bites and other systemic manifestations, suggesting that NKC lymphocytosis may show severe hypersensitivity to mosquito bites as the most outstanding manifestation[53]. In patients with primary biliary cirrhosis (a chronic, life-threatening disorder that is believed to be immunologically mediated), NKC function is low and poorly responsive to high concentration of IFN suggesting a kinetic impairment of NKC function in patients with PBC[54].

\section{NKC Activity in Experimental Allergic Encephalomyelitis}

A decrease in NKC activity was detected at the onset of clinically defined experimental allergic encephalomyelitis (EAE) disease in long-tailed macaques (Macaca fascicularis) by inoculation of autologous myelin basic protein (BP) in complete Freund's adjuvant[55]. There was a statistically significant lower NKC activity than normal controls in atopic patients when related to a low IL-2 production. Both facts inversely correlated with the concentration of IgE in serum. We concluded that atopic patients' vulnerability to fungal infections represent probably a defective NKC activity. Suppressor T-cell function is abnormal in these patients. Both defects could be due to a faculty immunoregulatory helper function.

\section{CONCLUSIONS}

Considering the dangers involved in attempting to make clinical evaluations based on results of isolated in vitro assessment of quantity or function of immune system cellular and humoral components, the medical history, physical examination, and objective neurological evaluations should be used as confounding variables that could affect the increase in the validity of the outcomes. In the light of this review, it is concluded that chronic exposures to toxigenic mold 
could lead to abnormal NKC activity with a wide range of neurological consequences some of which are headache, general debilitating pains, body temperature up to $40^{\circ} \mathrm{C}\left(104^{\circ} \mathrm{F}\right)$, cough, memory loss, depression, mood swings, sleep disturbances, anxiety, chronic fatigue, and seizures.

\section{ACKNOWLEGEMENT}

The authors are grateful to the mothers who participated in this study. Development of this paper was made possible through financial support from the Catherine Elliott Fund, Liverpool School of Tropical Medicine, Liverpool, England, United Kingdom.

\section{REFERENCES}

1. Lazarus, A.H. and Baines, M.G. (1985) Studies on the mechanism of specificity of human natural killer cells for tumor cells: correlation between target cell transferrin receptor expression and competitive activity. Cell Immunol. 96, 255.

2. MacDougall, S.L., Shustik, C., and Sullivan, A.K. (1986) Target cell specificity of human natural killer cells. II. Apparent change with activation. Cell Immunol. 103, 352.

3. Roitt, I. (1988) Essential Immunology, $6^{\text {th }}$ ed. Blackwell, Oxford.

4. Rodriguez-Calvillo, M., Duarte, M., Tirapu, I., Berraondo, P., Mazzolini, G., Qian, C., Prieto, J., and Melero, I. (2002) Upregulation of natural killer cells functions underlies the efficacy of intratumorally injected dendritic cells engineered to produce interleukin-12. Exp. Hematol. 30(3), 195-204.

5. Piccioli, D., Sbrana, S., Melandri, E., and Valiante, N.M. (2002) Contact-dependent stimulation and inhibition of dendritic cells by natural killer cells. J. Exp. Med. 195(3), 335-341.

6. Bahr, M.J. and Manns, M.P. (2001) Function of the immune system in liver cirrhosis. Z. Gastroenterol. 39(8), 601-607.

7. Ryan, J.C., Naper, C., Hayashi, S., and Daws, M.R. (2001) Physiologic functions of activating natural killer (NK) complex-encoded receptors on NK cells. Immunol. Rev. 181, 126-137.

8. Katz, P., Zaytoun, A.M., Lee, J.H., Panush, R.S., and Longley, S. (1982) Abnormal natural killer cell activity in systemic lupus erythematosus: an intrinsic defect in the lytic event. J. Immunol. 129(5), 19661971.

9. Katz, P., Zaytoun, A.M., and Lee, J.H. (1982) Mechanisms of human cell-mediated cytotoxicity. III. Dependence of natural killing on microtubule and microfilament integrity. J. Immunol. 129(6), 2816-2825.

10. Hsueh, C.M., Chen, S.F., Lin, R.J., and Chao, H.J. (2002) Cholinergic and serotonergic activities are required in triggering conditioned NK cell response. J. Neuroimmunol. 123(1-2), 102-111.

11. Miller, J.S. (2001) The biology of natural killer cells in cancer, infection, and pregnancy. Exp. Hematol. 29(10), 1157-1168.

12. Su, H.C., Nguyen, K.B., Salazar-Mather, T.P., Ruzel, M.C., Dalod, M.Y., and Biron, C.A. (2001) NK cell functions restrain T cell responses during viral infections. Eur. J. Immunol. 31(10), 3048-3055.

13. Thuvander, A., Breitholtz-Emanuelsson, A., and Olsen, M. (1995) Effects of ochratoxin A on the mouse immune system after subchronic exposure. Food Chem. Toxicol. 33(12), 1005-1011.

14. Luster, M.I., Germolec, D.R., Burleson, G.R., Jameson, C.W., Ackermann, M.F., Lamm, K.R., and Hayes, H.T. (1987) Elective immunosuppression in mice of natural killer cell activity by ochratoxin A. Cancer Res. 47(9), 2259-2263.

15. Reddy, R.V. and Sharma, R.P. (1989) Effects of aflatoxin B1 on murine lymphocytic functions. Toxicology 54(1), 31-44.

16. Thuvander, A., Funseth, E., Breitholtz-Emanuelsson, A., Hallen, I.P., and Oskarsson, A. (1996) Effects of ochratoxin A on the rat immune system after perinatal exposure. Nat. Toxins 4(3), 141-147.

17. Berek, L., Petri, I.B., Mesterhazy, A., Teren, J., and Molnar, J. (2001) Effects of mycotoxins on human immune functions in vitro. Toxicol. In Vitro 15(1), 25-30.

18. Berek, L., Petri, I.B., Mesterhazy, A., and Teren, J. (2002) The immunosuppressive effect of Fusarium mycotoxin as a function of HLA antigens. Acta Microbiol. Immunol. Hung. 49(1), 99-104.

19. Methenitou, G., Maravelias, C., Athanaselis, S., Dona, A., and Koutselinis, A. (2001) Immunomodulative effects of aflatoxins and selenium on human natural killer cells. Vet. Hum. Toxicol. 43(4), 232-234.

20. Liu, H., Jacman, S., Driscoll, H., and Larsen, B. (2000) Immunologic effects of gliotoxin in rats; mechanisms for prevention of autoimmmune diabetes mellitus. Ann. Clin. Lab. Sci. 30(4), 336-378.

21. Llewellyn, G.C., Mccay, J.A., Brown, R.D., Musgrove, D.L., Butterworth, L.F., Munson, A.E., and White, K.L., Jr. (1998) Immunological evaluation of the mycotoxin patulin in female B6C3F1 mice. Food Chem. 
Toxicol. 36(12), 1107-1115.

22. Yovel, G., Sirota, P., Mazeh, D., Shakhar, G., Rosenne, E., and Ben-Eliyahu, S. (2000) Higher natural killer cell activity in schizophrenic patients: the impact of serum factors, medication, and smoking. Brain Behav. Immun. 14(30), 153-169.

23. Salvaggio, J.E. (1996) Understanding clinical immunological testing in alleged chemically induced environment illnesses. Regul. Toxicol. Pharmacol. 24(1), S16-27.

24. Gersuk, G.M., Carmel, R., Pattamakom, S., Challita, P.M., Rabinowitz, A.P., and Pattengale, P.K. (1993) Quantitative and functional studies of impaired natural killer (NK) cells in patients with myelofibrosis, essential thrombocythemia, and polcythemia vera. I. A potential role for platelet-derived growth factor in defective NK cytotoxicity. Nat. Immun. 12(3), 136-151.

25. Ozturk, L., Pelin, Z., Karadeniz, D., Kaynak, H., Cakar, L., and Gozukirmizi, E. (1999) Effects of 48 hours of sleep deprivation on human immune profile. Sleep Res. Online 2(4), 107-111.

26. Heiser, P., Dickhaus, B., Schreiber, W., Clement, H.W., Hasse, C., Henning, J., Remschmidt, H., Krieg, J.C., Wesemann, W., and Opper, C. (2000) White blood cells and cortisol after sleep deprivation and recovery sleep in humans. Eur. Arch. Psychiatry Clin. Neurosci. 250(1), 16-23.

27. Masterson, B.J., Galask, R.P., and Ballas, Z.K. (1996) Natural killer cell function in woman with Vestibulitis. J. Reprod. Med. 41(8), 562-828.

28. Irwin, M., Mascovich, A., Gillin, J.C., Willoughby, R., Pike, J., and Smith, T.L. (1994) Partial sleep deprivation reduces natural killer cell activity in humans. Psychosom. Med. 56(6), 493-498.

29. Irwin, M., Smith, T.L., and Gillian, J.C. (1992) Electroencephalographic sleep and natural killer activity in depressed patients and control subjects. Psychosom. Med. 54(1), 10-21.

30. Irwin, M. (2001) Neuroimmunology of disordered sleep in depression and alcoholism. Neuropsychopharmacology 25(5 Suppl), S45-49.

31. Koga, C., Itoh, K., Aoki, M., Suefuji, Y., Yoshida, M., Asosina, S., Esaki, K., and Kameyama, T. (2001) Anxiety and pain suppress the natural killer cell activity in oral surgery outpatients. Oral Surg. Oral Med. Oral Pathol. Oral Radiol. Endod. 91(6), 654-658.

32. Logan, H.L., Lutgendorf, S., Kirchner, H.L., Rivera, E.M., and Lubaroff, D. (2001) Pain and immunologic response to root canal treatment and subsequent health outcomes. Psychosom. Med. 63(3), 453-462.

33. Greisen, J., Hokland, M., Grofte, T., Hansen, P.O., Jensen, T.S., Vilstrup, H., and Tonnesen, E. (1999) Acute pain induces an instant increase in natural killer cell cytotoxicity in humans and this response is abolished by local anaesthesia. Br. J. Anaesth. 83(2), 235-240.

34. Gelderblom, W.C., Marasas, W.F., Lebepe-Mazur, S., Swanevelder, S., Vessey, C.J., and Hall, P.L. (2002) Interaction of fumonisin B (1) and aflatoxin B (1) in a short-term carcinogenesis model in rat liver. Toxicology 171(2-3), 161-173.

35. Stettler, P.M. and Sengstag, C. (201) Liver carcinogen aflatoxin B1 as an inducer of mitotic recombination in a human cell line. Mol. Carcinog. 31(3), 125-138.

36. Stettler, P.M. and Sengstag, C. (2001) Liver carcinogen aflatoxin B1 as an inducer of mitotic recombination in a human cell line. Mol. Carcinog. 31(3), 125-138.

37. Kodell, R.L., Young, J.F., Delongchamp, R.R., Turturro, A., Chen, J.J., Gaylor, D.W., Howard, P.C., and Zheng, Q. (2001) A mechanistic approach to modeling the risk of liver tumours in mice exposed to fumonisin B1 in the diet. Food Addit. Contam. 18(3), 237-253.

38. Morikawa, S., Naito, M., and Takamizawa, H. (1986) Effects of neonatal androgenization on endometrial carcinogenesis and natural killer (NK) activity. Nippon Sanka Fujinka Gakkai Zasshi 38(11), 1957-1963.

39. Baumann, M.A., Milson, T.J., Patrick, C.W., Libnoch, J.A., and Keller, R.H. (1986) Correlation of circulating natural killer cell count with prognosis in large cell lymphoma. Cancer 57(12), 2309-2312.

40. Komiyama, A., Kawai, H., Yamada, S., Aoyama, K., Yamazaki, M., Saitoh, H., Miyagawa Y, Akabane T, and Uehara Y. (1985) Impaired natural killer cell recycling in childhood chronic neutropenia with morphological abnormalities and defective chemotaxis of neutrophils. Blood 66(1), 99-105.

41. James, S.P. and Jones, E.A. (1985) Abnormal natural killer cytotoxicity in primary biliary cirrhosis: evidence for a functional deficiency of cytolytic effector cells. Gastroenterology 89(1), 165-171.

42. Umiel, T., Friedman, E., Luria, D., Cohen, I.J., Kaplinssky, H., Netzer, L., Pecht, M., Trainin, N., and Zaizov, R. (1984) Impaired immune regulation in children and adolescents with hemophilia and thalassemia in Israel. Am. J. Pediatr. Hematol. Oncol. 6(4), 371-378.

43. O’Brien, E., Heussner, A.H., and Dietrich, D.R. (2001) Species-, sex-, and cell type-specific effects of ochratoxin A and B. Toxicol. Sci. 63(2), 256-264.

44. Ramljak, D., Calvert, R.J., Wiesenfeld, P.W., Diwan, B.A., Catipovic, B., Marasas, W.F., Victor, T.C., Anderson, L.M., and Gelderblom, W.C. (2000) A potential mechanism for fumonisin B (1)-medicated hepatocarcinogenesis: cyclin D1 stabilization associated with activation of Akt and inhibition of GSK-3beta activity. Carcinogenesis 21(8), 1537-1546.

45. Gelderblom, W.C., Abel, S., Smuts, C.M., Swanevelder, S., and Snyman, S.D. (1999) Regulation of fatty acid biosynthesis as a possible mechanism for the mitoinhibitory effect of fumonisin B1 in primary rat hepatocytes. Prostaglandins Leukot. Essent. Fatty Acids 61(4), 225-234. 
46. Yeung, J.M., Wang, H.Y., and Prelusky, D.B. (1966) Fumonisin B1 induces protein kinase C translocation via direct interaction with diacylglycerol binding site. Toxicol. Appl. Pharmacol. 141(1), 178-184.

47. Mistry, K.J., Krishna, M., Pasupathy, K., Murthy, V., and Bhattacharya, R.K. (1996) Signal transduction mechanism in response to aflatoxin B1 exposure: protein kinase $\mathrm{C}$ activity. Chem. Biol. Interact. 100(2), 177-185.

48. Lin, C.W., Parker, H.M., Vesonder, R.F., and Haschek, W.M. (1996) Intravenous fumonisin B1 induces cell proliferation and apoptosis in the rat. Nat. Toxins 4(1), 34-41.

49. Schroeder, J.J., Crane, H.M., Xia, J., Liotta, D.C., and Merrill, A.H., Jr. (1994) Disruption of sphingolipid metabolism and stimulation of DNA synthesis by fumonisin B1. A molecular mechanism for carcinogenesis associated with Fusarium moniliforme. J. Biol. Chem. 269(5), 3475-3481.

50. Uchida, A. (1992) Chronic fatigue immune dysfunction syndrome. Nippon Rinsho 50(11), 2625-2629.

51. Richards, S.J. and Scott, C.S. (1992) Human NK cells in health and disease: clinical, functional, phenotypic and DNA genotypic characteristics. Leuk. Lymphoma 7(5-6), 377-399.

52. Tsukada, T., Ohno, T., Morita, K., Otsuji, H., Sekine, T., Nishikawa, M., Kobayashi, T., Kita, K., Shirakawa, S., and Namba, K. (1991) Aggressive natural killer cell lymphoproliferative disease of large granular lymphocytes with leukemia-like clinical course in the terminal stage. Rinsho Ketsueki 32(2), 162166.

53. Tokura, Y., Tamura, Y., Takigawa, M., Koide, M., Satoh, T., Sakamoto, T., Horiguchi, D., and Yamada, M. (1990) Severe hypersensitivity to mosquito bites associated with natural killer cell lymphocytois. Arch. Dermatol. 126(3), 362-368.

54. Matheson, D.S., Green, B.J., and Minuk, G.Y. (1989) Natural killer cell activity and the response to interferons alpha, beta, and gamma in patients with primary biliary cirrhosis. J. Allergy Clin. Immunol. 84(2), 214-218.

55. Rose, L.M., Clark, E.A., Hruby, S., and Alvord, E.C., Jr. (1987) Fluctuations of T-and B-cell subsets in basic protein-induced experimental allergic enecphalomyelitis (EAE) in long-tailed macaques. Clin. Immunol. Immunopathol. 44(1), 93-106.

56. Frajman, M., Gonzalez, L., Alvarado, A., and Yock, J. (1987) Cellular immunity and IgE levels in atopic patients. Allergy 42(2), 81-84.

This article should be referenced as follows:

Anyanwu, E.C., Campbell, A.W., Jones, J., and Ehiri, J. (2003) The neurological significance of abnormal natural killer cell activity in chronic toxigenic mold exposures. TheScientificWorldJOURNAL 3, 1128-1137.

Handling Editor:

Joav Merrick, Principal Editor for Child Health and Human Development — a domain of TheScientificWorldJOURNAL.

\section{BIOSKETCHES}

Ebere C. Anyanwu, Ph.D., is a Clinical Neurophysiologist and the Director of Cahers Neurosciences Research Inc., Houston, TX. He is also an Adjunct Professor of Anatomy and Physiology at the North Harris and Montgomery Colleges in Houston and the Woodlands, TX.

Andrew W. Campbell, M.D., is an Expert Medical Scientist in Immunotoxicology and the Medical Director and Chief Executive of the Center for Immune, Environmental, and Toxic Disorders, Houston, TX. E-mail: md@immunotoxicology.com.

Joseph Jones, Ph.D., is the Dean of Graduate School and Associate Provost for Research, Texas Southern University, Houston. E-mail: jones_jx@tsu.edu.

John Ehiri, Ph.D., is an Assistant Professor in the Department ofMaternal and Child Health, School of Public Health, University of Alabama at Birmingham, USA. E-mail: jehiri@uab.edu. 

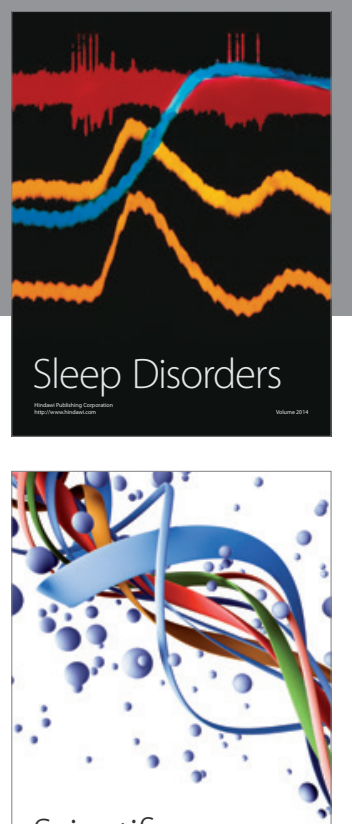

Scientifica
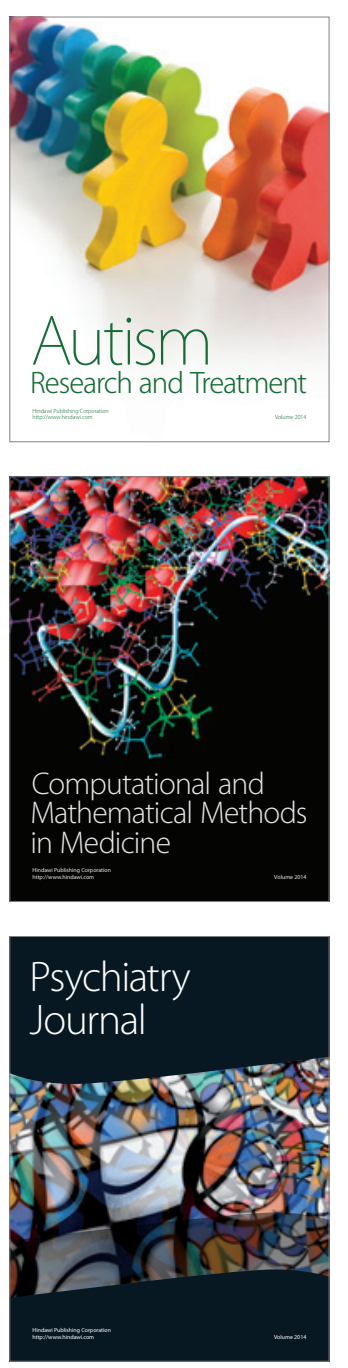
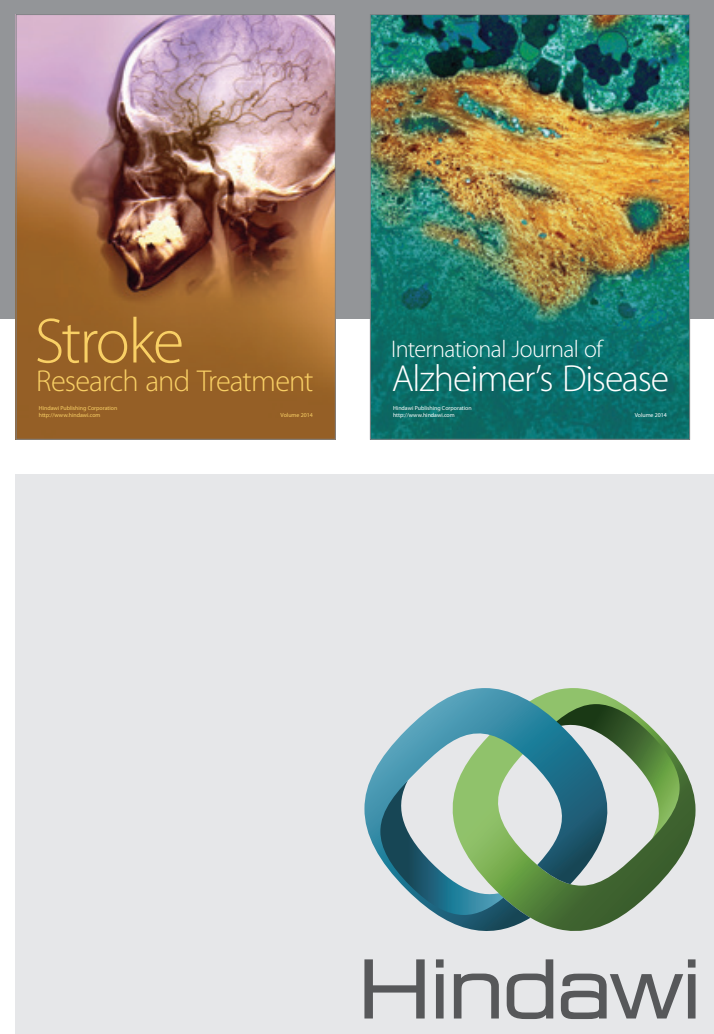

Submit your manuscripts at

http://www.hindawi.com
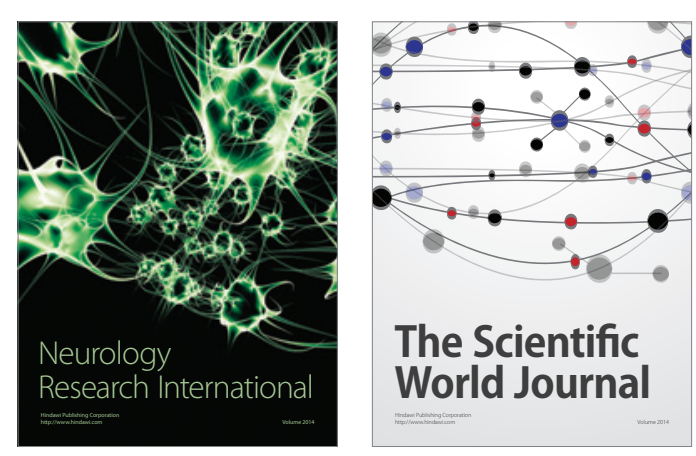

The Scientific World Journal

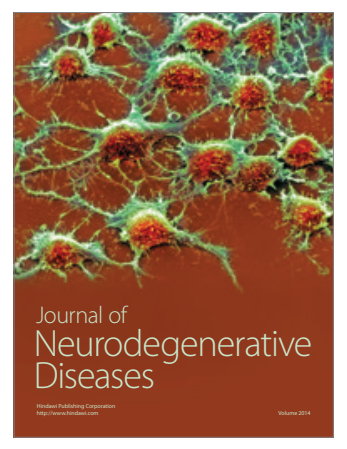

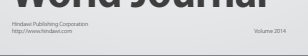

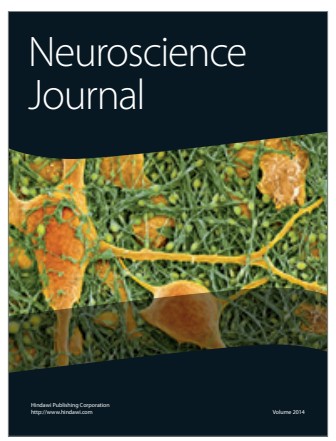

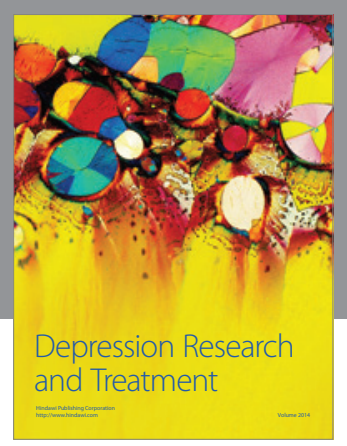
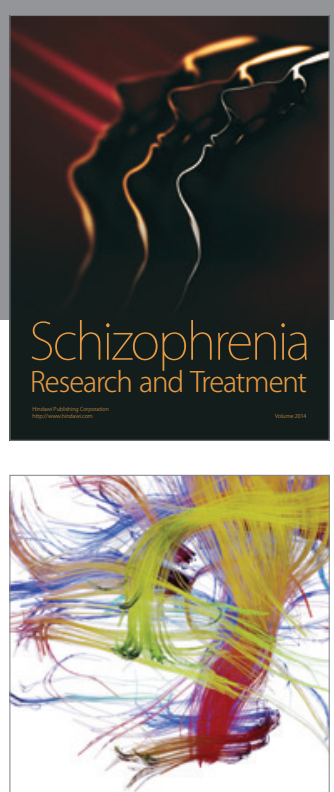

Brain Science

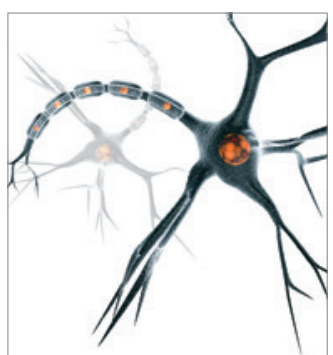

Neural Plasticity
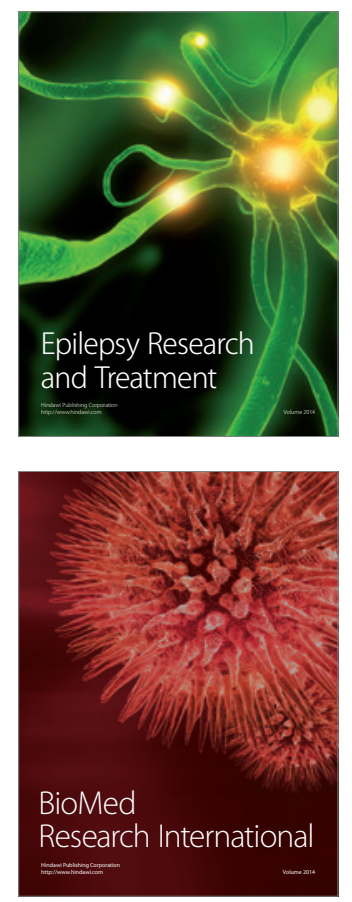

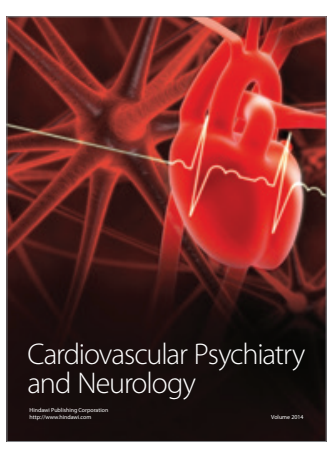

Parkinson's

Disease
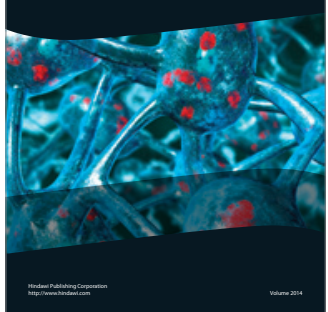